\title{
Adapting to the "New Normal" in Orthopaedic Trauma During COVID-19
}

\section{Dear Editor,}

COVID-19 hit Singapore in January 2020 and by February, the health ministry had raised the Disease Outbreak Response System Condition (DORSCON) alert levels to orange. ${ }^{1}$ Multiple control measures were implemented, and hospital resources were redirected to the frontline, reserving limited capacity to treating emergencies. ${ }^{2,3}$ One of the key units preserved within Tan Tock Seng Hospital (TTSH) was the trauma unit. Treatment of life threatening (Priority 1) trauma cases continued, whereas other cases were mostly diverted within the local trauma network. In orthopaedics, electives were cancelled whilst the trauma service continued, tending to the fractures that still occurred. Every hospital developed detailed business-continuity plans, placing unique challenges to orthopaedic trauma services. ${ }^{4,5}$ We expounded on the mitigating actions at a Level 1 Trauma Centre in Singapore and described the strategies used to handle the "new normal".

\section{Impact on Orthopaedic Trauma}

Emergency department (ED) attendances soared at our institution, being annexed to National Centre for Infectious Diseases (NCID) - a purpose-built facility to handle infectious disease cases in a self-contained manner, with isolation and intensive care wards, operating theatres (OTs), radiology, and laboratory facilities, as well as a screening centre-which handled about $50-70 \%$ of the screening load for COVID-19 in Singapore. ${ }^{5}$ Although our unit was meant to be preserved, we were also redeployed to the screening centre during this surge. This was part of our institutional efforts to ensure sufficient capacity to handle COVID-inpatient care, based on the previous experiences during SARS outbreak. ${ }^{6}$

Specific measures were taken to maintain the availability of the orthopaedic trauma surgeon for managing complex trauma. We divided the unit into Team A and B, each comprising a consultant, an associate consultant and 2 residents, who avoided close contact with one another. On trauma rosters, a consultant was paired with a resident, forming sub-teams that ran trauma lists and clinic sessions together. With the shortage in house-officers, we assigned them to the wards, and they communicated with the teams via clear clinical documentation supplemented by telecommunication. This allowed the house-officers to avoid close contact with the unit. Furthermore, arrangements were also made for manpower diversion from outpatient clinics if mass quarantine happened. We staggered the NCID deployment with 1 member committed at a time, and always ensured an available trauma consultant. Deliberate arrangements were made for residents from other services to cross-cover the trauma lists, which was critical for the continued $\mathrm{s}$ ervice of the unit. However, this meant that frequent handing-over-taking-over was required, and communication between team members became key. Clear documentation of decisions was emphasised, with specific instructions for recording the rationale behind clinical decisions. Covering consultants made decisions for another consultant's patients during his/her absence, including outpatient followup visits. Communications were supplemented with secured text-messaging systems, teleconversations, and clinical photographs where appropriate, minimising any potential lapses in continuity of care. These were essential for trauma patients, given the highly variable soft tissue conditions that were heavily influenced patient outcomes.

Confirmed/suspect cases and pneumonia cases with unknown status were operated in designated OTs to avoid contamination of trauma lists reserved for other patients. Polytrauma cases were handled within the main building where access to multidisciplinary trauma support was quicker, and made sense for unstable patients who were often sent to the main building resuscitation bays at first instance (Fig. 1A). The time needed for preparation was lengthy, with decontamination procedures disrupting normal operations for substantial periods. These patients 


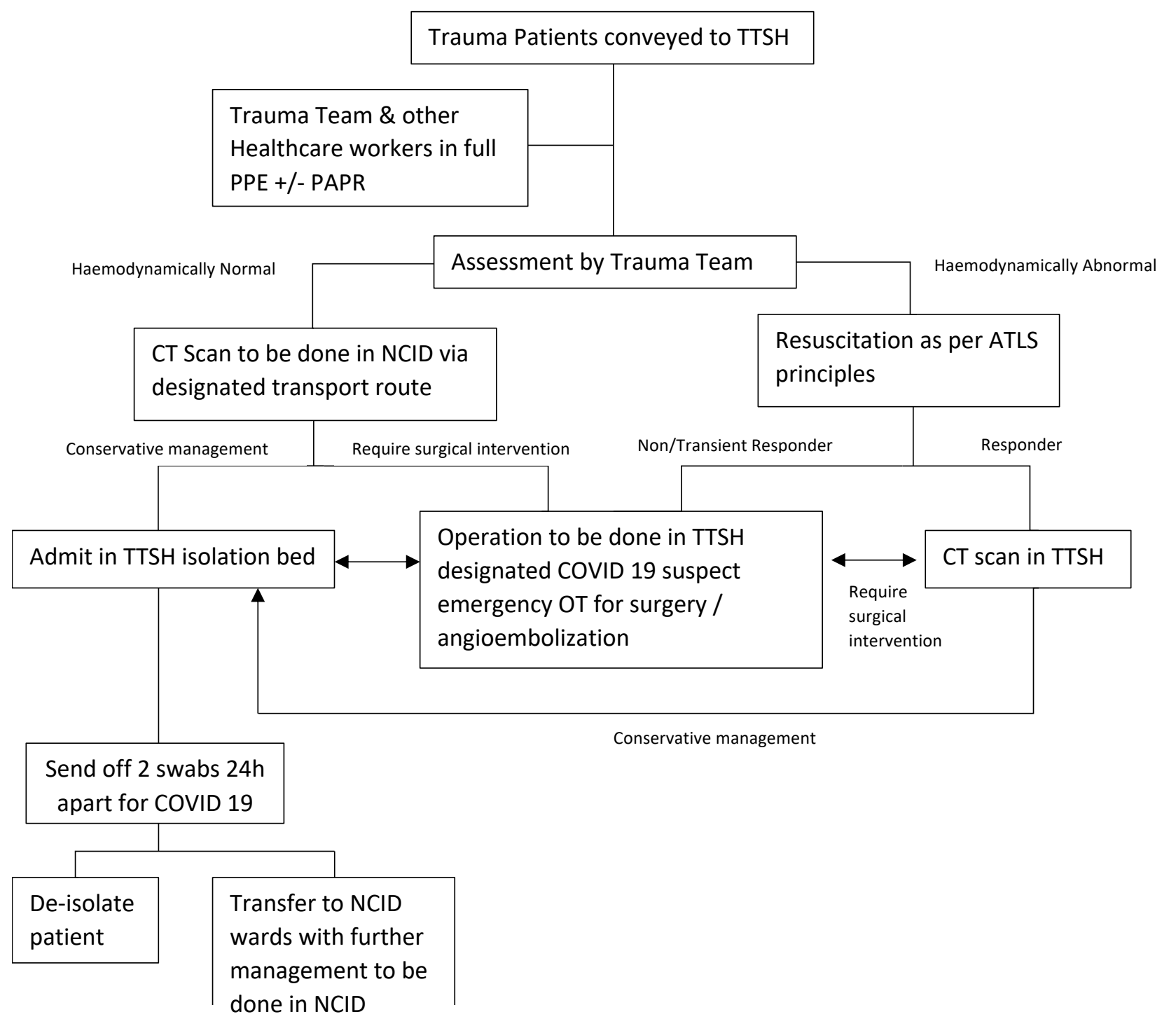

Figure 1A. Management Workflow of Trauma Patients Conveyed to TTSH

ATLS: Advanced trauma life support; CT: Computed tomography; NCID: National centre for infectious diseases; OT: Operating theatre; PPE: Personal protective equipment; PAPR: Powered air-purifying respirator

*Acknowledgement: Dr Teo Li-Tserng, Director of Trauma, TTSH Trauma Service, is the workflow process owner. 
needed to go straight into the OTs, hence the pathways en-route had to be cleared prior to transfers. Detailed coordination was done using nursing manpower freed up from elective theatres. Once a trauma-activation case was known, other OTs ceased operations. Separate workflows were present for patients transferred to NCID resuscitation bays (Fig. 1B). The NCID-OTs were utilised for stable cases admitted to NCID wards. In total, we operated on five COVID-19-suspect patients there. They were migrant workers and one was transferred from a hospital in Batam, Indonesia. These OTs were well equipped with negative pressure airflow systems and high efficiency particulate filters, optimal for reducing transmission risks. All healthcare workers wore personal protective equipment throughout the surgeries, and the anaesthetists used powered air-purifying respirators (PAPR) for airway intubations. Surgeries were performed expediently, and selected equipment were brought into the operating room to reduce the post-procedural cleaning required. Electrocautery usage was kept to a minimum as a precautionary measure, and the number of personnel within the operating room was also minimised. ${ }^{7}$

\section{Adapting to the "New Normal"}

\section{Clinical Decision-making and Patient Care}

From January to April, there was a drastic reduction in orthopaedic trauma caseload. We saw a $20-25 \%$ decrease in operative caseload during the early phases, and when strict measures during "Circuit Breaker" were introduced, there was a further decrease of about $90 \%$ (Fig. 2). ${ }^{1}$ Notably, there was a reduction in geriatric hip fractures, trauma from road traffic accidents and sporting injuries. The changes in caseload and case-mix were in part due to social distancing, with less people leaving their residences, less travel on the roads, and no injuries from contact sports (activities that still took place were jogging and cycling). More importantly, other hospitals in our local trauma network had off-loaded the non-urgent trauma cases during this period, so that TTSH could redivert manpower to maintain operations at the NCID. Such a whole of healthcare response is unique to Singapore. As a result, we saw an unexpected decrease in hip fractures which tended to occur at home. There will likely be peaks and troughs in the coming months, with operative load increasing with relaxing of measures and dipping once measures kick in when

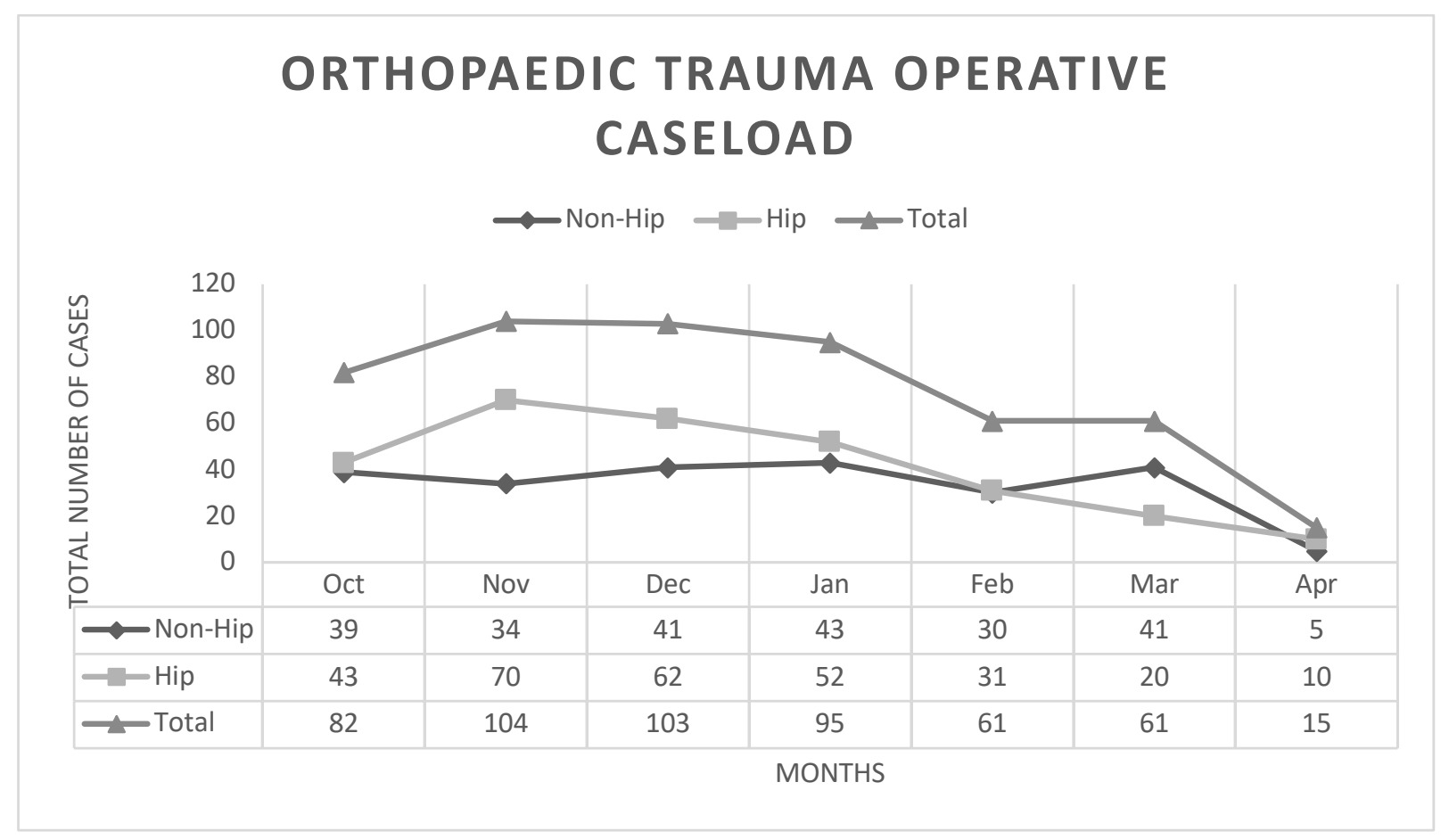

Figure 2: Orthopaedic trauma monthly caseload numbers on semi-elective operative list. 
COVID-19 cases spike. Regardless, our unit is prepared for the "new normal", ready to accelerate through the gears according to the demands.

Holistic treatment decisions were made to minimise "face-to-face" encounters. As an overarching principle, risk/benefit ratios were considered in accordance with the health, age, and co-morbidities of each patient. ${ }^{8,9}$ We prioritised surgeries based on their indications. Surgeries were performed in those with clear indications. In those without, surgeries were deferred if there were no social or functional demands arising from social distancing requirements that could be addressed via surgical treatment. Therefore, in cases where previously patient choice determined treatment, surgeries were deferred after discussions about the risks related to disease transmission vis-a-vis fracture sequela from delayed fracture repair (e.g. proximal humeral fractures and clavicle fractures). However, surgical treatment afforded a quicker return to independence. This was evidenced in uncomplicated fixations of humeral shaft fractures, for the otherwise healthy individual staying alone, restoring independence in activities such as dressing and feeding right after surgery. Unique to this pandemic, surgical treatment of such fractures possibly did our patients more favours compared to conventional wisdom of treating these conservatively. Treating cases conservatively resulted in reduced inpatient stays but an increase in outpatient visits. According to our experience, casting of fractures required closer follow-ups, but the visit frequencies were not more than operative cases, which required wound checks, assessment of bone healing and limb functioning.

It must be emphasised that we must continue to respect the indications for surgery and provide sound advice when needed. The principles adhered to are: (1) close monitoring to determine if early surgery is needed; (2) if surgery becomes indicated, admit for fracture repair or reconstruction emergently; (3) for late presentation or development of post-traumatic deformity, aim to maximise function while awaiting available resource for reconstruction. For example, in cases without absolute surgical indications, conservative and surgical treatment for proximal humeral fractures have similar published outcomes. ${ }^{10}$ Hence, we treated these cases conservatively and monitored for fracture sequela. Should the indications for surgery arise, they will be evaluated for suitability of early osteosynthesis, contingent on fracture factors such as adequacy in bone stock for anatomical restoration, posteromedial cortical support, rotator cuff integrity and joint stability, and patient factors such as degree of osteoporosis, smoking and comorbidities. Arthroplasty options will also be considered. ${ }^{11}$ However, fractures that do not have as many options in the delayed setting, with known evidence-based outcomes, will not be amenable to this approach.

\section{Surgical Priorities}

The increase in reconstructive surgeries will be juxtaposed with the demand for elective surgeries (previously cancelled at the pack of the outbreak) when the pandemic abates. Like when the crisis first started, orthopaedic departments will again need to agree to certain rules of prioritisation. Should trauma cases take priority given that reconstructive surgeries have long surgical times and less predictable outcomes? Or should quick and efficient elective sports and adult reconstructive surgeries take priority as the outcomes are predictable and considerably more "cost effective"? When COVID-19 started, our institution prioritised operative resources for "urgent" cases, and when it tails off, we will continue the modus operandi. However, this will evolve to include "urgency" from a risk management perspective for electives. Priority will be given to patients whose condition has aggravated, followed by those whose surgeries had been postponed multiple times. Next will be cases of fracture sequela after initial conservative treatment and patients who had earlier deferred their surgeries. Given the competing demands, it will be essential to ensure adequate manpower provision as this will coincide with increased outpatient attendances. Therefore, we will need to scrutinise referrals and continue proactive appointment management even after the peak of the pandemic is over-necessarily so to sustain the already weary workforce and maintain quality trauma care, till a time when normalcy can be safely restored.

\section{Conclusion}

COVID-19 led to a drastic reduction of surgical caseload for orthopaedic trauma and posed unique challenges to the trauma surgeon. Significant efforts were made to minimise impact on patient care. The longer-term impact it has on patient outcomes remains to be seen, but we must be prepared to offer the best care for our patients. 


\section{REFERENCES}

1. Singapore Ministry of Health. Risk assessment raised to disease outbreak response system condition (DORSCON) Orange. 2020 Feb 7. Accessed 2020 Mar 13. https://www.moh.gov.sg/news-highlights/ details/risk-assessment-raised-to-dorscon-orang

2. Liang ZC, Wang W, Murphy D, Hui JHP. Novel Coronavirus and Orthopaedic Surgery: Early Experiences from Singapore J Bone Joint Surg Am 2020 May 6;102:745-749.

3. Goh KJ, Choong MC, Cheong EH, Kalimuddin S, Duu Wen S, Phua GC et al. Rapid Progression to Acute Respiratory Distress Syndrome: Review of Current Understanding of Critical Illness from COVID-19 Infection. Ann Acad Med Singapore 2020;49:108-118.

4. Liow MH, Tay KXK, Yeo NEM, Tay DKJ, Goh SK, Koh JSB et al. Ensuring Business Continuity of Musculoskeletal Care During the COVID-19 Pandemic. JBJS Open Access 2020;5:e0050

5. Soh TLT, Ho SWL, Yap WMQ, Oh JY. Spine Surgery and COVID-19: Challenges and Strategies from the Front Lines. J Bone Joint Surg Am 2020;102:e56.

6. Tai DY. SARS: how to manage future outbreaks? Ann Acad Med Singapore 2006;35:368-373.

7. Iorio-Morin C, Hodaie M, Sarica C, Dea N, Westwick HJ, Christie SD et al. Letter: The Risk of COVID-19 Infection During Neurosurgical Procedures: A Review of Severe Acute Respiratory Distress Syndrome Coronavirus 2 (SARS-CoV-2) Modes of Transmission and Proposed Neurosurgery-Specific Measures for Mitigation. Neurosurgery 2020;87(2):E178-E185.

8. Chen N, Zhou M, Dong X, Qu J, Gong F, Han Y et al. Epidemiological and clinical characteristics of 99 cases of 2019 novel coronavirus pneumonia in Wuhan, China: a descriptive study. Lancet 2020;395(10223):507-513.

9. Zhou F, Yu T, Du R, Fan G, Liu Y, Liu Z et al. Clinical course and risk factors for mortality of adult inpatients with COVID-19 in Wuhan, China: a retrospective cohort study. Lancet 2020; 395:1054-1062.

10. Rangan A, Handoll H, Brealey S, Jefferson L, Keding A, Martin BC et al. Surgical vs nonsurgical treatment of adults with displaced fractures of the proximal humerus: the PROFHER randomized clinical trial. JAMA 2015;313:1037-1047.

11. Mansat P, Bonnevialle N. Treatment of fracture sequelae of the proximal humerus: anatomical vs reverse shoulder prosthesis. Int Orthop. 2015;39(2):349-354.

Junren Zhang, ${ }^{1} M M E D$ (Ortho), FRCS (Ortho), FAMS,

Michael Yam, ${ }^{1} M M E D$ (Ortho), MRCS (Edin), FRCS (Ortho),

Ivan TH Chua, ${ }^{1} M M E D$ (Ortho), MRCS (Edin), FRCS (Ortho)

${ }^{1}$ Department of Orthopaedic Surgery, Tan Tock Seng Hospital, Singapore

Address for Correspondence: Dr Ivan Tjun Huat Chua, Department of Orthopaedic Surgery, Tan Tock Seng Hospital, Jalan Tan Tock Seng, Singapore 308433

Email: ivan_chua@ttsh.com.sg 